MADAN : THE COLLOID FORM OF PIPERINE.

\title{
XCVII.-The colloid form of Piperine, with especial reference to its Refractive and Dispersive Powers.
}

\section{By Henry G. MAdan, M.A.}

Piperine, the alkaloid extracted from pepper by digestion with alcohol, appears to have attracted what little attention has been bestowed upon it rather from its chemical than from its physical properties. Wackenroder, however (quoted by Gmelin, Handbook of Chemistry, 15, 18*), observed that the crystallised substance, after being melted, solidified on cooling into a resinous, strongly refracting mass. More recently, this resinous form of piperine has been tried as a material for mounting objects for examination under the microscope, but it has not been found satisfactory, partly from an apparent want of permanency, but mainly because it is found to interfere with the definition of objects immersed in it. The reason of this will be evident from what is said in the latter part of this paper with reference to its dispersive power.

It seemed desirable, in the first place, to ascertain the exact conditions under which crystalloid piperine is converted into colloid piperine, and vice versa. For this purpose, some of the ordinary substance, crystallised in monoclinic prisms, was placed in a beaker, and the latter was placed in an oil-bath maintained at a temperature of $180^{\circ}$. The crystals melted at $132^{\circ}$, and as soon as the whole was fully liquefied a portion of it was poured off upon a glass plate and marked "A." The beaker was replaced in the oil-bath and further heated. As soon as its contents had reached the temperature of the bath, namely, $180^{\circ}$, they were poured off upon another glass plate and labelled "B."

In both cases, $A$ and $B$, the substance solidified to a light yellow, transparent resin (the colour of $B$ being rather darker than that of $A$ ), without any sign of crystallisation. When cold, the mass was tough and slightly "tacky," receiving a faint indentation from the fingernail. A small portion was detached from specimen $A$ and heated slowly on a water-bath. At a temperature just below $100^{\circ}$ it quickly

* The original paper appeared in Archiv. des Apothekerverein im nördlichen Deutsch. land, von Rud. Brandes, 37, 349 ; a reference which I have not been able tu verify. 
lost its transparency, becoming a light yellow, nearly opaque mass resembling sulphur, which under the microscope was seen to consist of a network of interlacing crystals. This, when further heated, underwent no apparent change until the usual melting point, $132^{\circ}$, was reached, when it fused in the ordinary way and solidified as before into a transparent resin on cooling. The process of reheating and cooling was repeated more than once, with the same results.

A portion detached from specimen $\mathrm{I} 3$ was treated similarly and behaved in a precisely similar way.

The remainders of the preparations $A$ and $B$ were set aside under a bell-jar and examined from time to time. In about ten days, yellow, sulphur-like spots began to appear on the surface of $\mathbf{A}$, and a little later in the interior of the mass also. These, when examined under the microscope, were found to consist of tufts of prismatic crystals, forming a very beautiful object in polarised light. They gradually extended, with the ultimate result that in about three months the surface was covered, and in about six months the whole mass had reverted to the crystalline condition.

With regard to preparation $B$, the same gradual change took place; perhaps rather more tardily than in the case of $A$, but with the same ultimate result.

Several similar sets of experiments have been made, which have only served to confirm the above observations.

It would appear, therefore, (1) that at a temperature only slightly, if at all, above its melting point crystalloid piperine is converted (for the most part, at any rate) into the colloid condition; (2) that the latter condition, when thus prepared, is not permanent, but reverts spontaneously, after the lapse of a moderate time, and quickly when exposed to a temperature of $100^{\circ}$, to the crystalloid form.

The closely analogous behaviour of sulphur will be at once recalled to mind. It is well known that octahedral sulphur $\left(\mathbf{S}_{a}\right)$, when heated to a temperature of $160^{\circ}$ or higher, changes into the colloid form known as "plastic sulphur," or " $\mathrm{S}_{y}$ " and that the latter, although it can be retained as such for a time by rapid cooling, reverts spontaneously in the course of a few days, and quickly when heated to a temperature a little below the usual melting point, to the original crystalloid form, $\mathrm{S}_{\alpha}$. A further important point bearing on these changes was observed by Frankenheim (J.pr. Chem., 1840, 16, 7), and also by Deville (Ann. Chim. Phys., 1856, [iii], 47, 111), namely, that during the heating of sulphur, at a certain point (or, perhaps, more than one point) of temperature a large amount of kinetic energy, supplied in the form of heat, is transferred to the sulphur molecules, in which it is stored up as some form of statical energy essential to the existence of the plastic condition. (In the phraseology of the 
older physicists, "a large amount of heat becomes latent.") This energy reappears in a kinetic form (that of heat) when $S_{\gamma}$ changes back into $S_{\alpha}$. To quote one proof of the latter fact, Deville, experimenting with a mass of a kilogram or more of sulphur heated to $200^{\circ}$, and allowed to cool under uniform conditions, found that while the mass cooled from $170^{\circ}$ to $165^{\circ}$ in 50 seconds, and from $140^{\circ}$ to $135^{\circ}$ in 70 seconds, it took no less than 104 seconds to cool from $155^{\circ}$ to $150^{\circ}$, and 125 seconds to cool from $150^{\circ}$ to $145^{\circ}$, the endothermic supply serving to arrest the fall in temperature due to loss of heat by radiation and convection. Similarly, when a mass of sulphur was exposed to a uniform source of heat, the temperature did not rise at a uniform rate, but slackened while the transfer of energy to the molecules was taking place.

Now, it seemed very probable that something of the same kind might occur in the case of piperine, and that a prolonged continuance of the supply of heat might be necessary to ensure the complete conversion of the crystalloid into the colloid condition. The following experiments were made with the view of ascertaining whether this was the case.

Some crystallised piperine was placed in a beaker immersed in an oil-bath maintained at a constant temperature of $180^{\circ}$. When the liquefied substance had attained the temperature of the bath, a small portion was poured off on to a glass plate, labelled " $\mathrm{C}$," and reserved for comparison. The remainder was maintained at $180^{\circ}$ for one hour, near the end of which time it became slightly viscous. The colour also darkened to a full yellow (orange in thick layers), probably owing to the separation of a trace of carbon at the high temperature, since at $200^{\circ}$ a perceptible amount of decomposition takes place. At the expiration of an hour, the whole was poured off and allowed to cool on a plate labelled "D." When it was cold, a small piece was broken off and gradually heated on a slip of glass. It did not crystallise at $100^{\circ}$, nor did it undergo any change until the usual melting point $\left(132^{\circ}\right)$ was reached, when it became viscous, but did not attain sufficient fluidity to be poured out until the temperature reached $170^{\circ}$ or nearly so. It was allowed to cool and again heated, and this was repeated three or four times, but not a trace of crystallisation was observed. A portion of the preparation "C," when treated in the same way, became crystalline at $100^{\circ}$, like the preparation "B" already described. The remainders of both preparations, " $C$ " and " $D$," were kept under a bell-jar and examined from time to time.

These experiments were made in October, 1898, and in about a fortnight " $C$ " was spotted with yellow; it passed altogether into the crystalline condition in about the usual time-six months. No change whatever was noticed in preparation " $D$," although its surface 
was scratched to promote crystallisation if possible; and up to the present time, more than two and a-half years since its preparation, it has remained quite unaltered, closely resembling ordinary "rosin" (colophony) in appearance, but not quite so brittle.

The inference which we seem entitled to draw from the results of the above experiments (and other similar ones, which corroborate them) is, that in order to ensure with even approximate certainty the conversion of crystalloid piperine into colloid piperine, an exposure to a temperature of not much less than $180^{\circ}$ for a period of not much less than one hour is required. Whether even then the tendency to revert to the crystalline condition is entirely overcome, time alone can show.

I may mention that I have made similar experiments with quinidine, and found it to behave in a similar way. It passes, when heated for an hour to $180^{\circ}$, into a permanent (as far as hitherto observed) colloid, having a rather high refractivity $\left(\mu_{D}=1.602\right)$.

\section{The Refractive and Dispersive Powers of Piperine.}

For the purpose of determining these, a hollow prism was made by cementing thin glass plates (selected with parallel surfaces) to the sides of a prism of dense flint glass. The height of the glass plates was rather more than twice that of the prism, so that they formed above the latter a cell of about the same size and having the same angles. This cell was filled with melted piperine, and thus a double prism was obtained, the lower part of dense flint glass and the upper part of piperine, with a refracting angle common to both. This was mounted in a brass frame with levelling screws for adjustment on the table of the spectrometer.

In the first place, the refracting angle was determined in the usual way; the mean of several concordant sets of readings giving $a=$ $24^{\circ} 10^{\prime} 10^{\prime \prime}$. Then the angle of minimum deviation $(\delta)$ for each of the principal lines of the spectrum was observed, both for the piperine prism and the glass one below it, and the corresponding indices of refraction were calculated by the usual formula,

$$
\mu=\sin \frac{1}{2}(\alpha+\delta) / \sin \frac{1}{2} \alpha .
$$

The results are given in the table on p. 926 .

It will be evident from the figures given in the table that colloid piperine possesses a higher refractivity than most, perhaps all, other resins or resin-like substances, the only one which comes at all near it, so far as I know, being naphthyl phenyl ketone (Phil. Mag., 1886, $[\nabla], 21,6245$ ) (for which $\mu_{D}=1.669$ ) and the compound which it forms with bromine. 
Indices of refraction of piperine and of dense fint glass.

\begin{tabular}{|c|c|c|}
\hline Spectrum-line. & Piperine. & Glass. \\
\hline 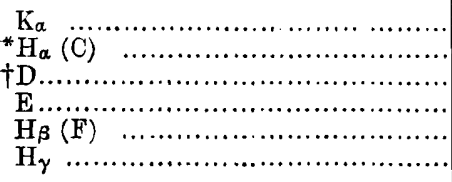 & $\begin{array}{l}1 \cdot 631 \\
1 \cdot 667 \\
1 \cdot 684 \\
1 \cdot 710 \\
1 \cdot 738 \\
1 \cdot 810\end{array}$ & $\begin{array}{l}1 \cdot 758 \\
1 \cdot 766 \\
1 \cdot 774 \\
1 \cdot 784 \\
1 \cdot 795 \\
1 \cdot 806\end{array}$ \\
\hline Coefficient of dispersion $\left(\mu_{\mathrm{H} \gamma}-\mu_{\mathrm{H} \alpha}\right)$ & $0 \cdot 143$ & 0.040 \\
\hline
\end{tabular}

* For the observation of the hydrogen-lines, one of the "end on" vacuum tubes devised by Prof. Piazzi Smyth was used, in which the light from a considerable thickness of glowing hydrogen is passed through the slit, and a very brilliant spectrum is obtained.

$\dagger$ The only other determination of the refractivity of piperine which I have been able to find is one by Dr. P. Riedel of Jena, quoted in Behrens' "Tabellen," (1st edition); and he only gives one index, namely, $\mu_{\mathrm{D}}=1.6844$, an agreement sufficiently close to promote confidence in my own results.

The above determinations were made with piperine which had not been heated for the full period of an hour. From experiments very recently made, it would seem that prolonged heating perceptibly diminishes the refractivity of the material.

The dispersive power of piperine is still more remarkable, being probably as great as, if not greater than, that of any other known substance. The coefficients of dispersion (taken as $\mu_{H \gamma}-\mu_{H a}$ ) of a few substances are as follows :

Piperine ................... $0 \cdot 14$

Phosphorus ................ 0.106

Methylene diiodide ......... 0.062

Phenylthiocarbimide ...... 0.060

Carbon disulphide ........ 0.057 $\alpha$-Monobromonaphthalene $\quad 0.051$ Dense flint glass $\ldots . . . . . \quad 0.040$

and it is obvious that piperine is far ahead of the others.

The diagram given on p. 927 shows the extent of the piperine spectrum as compared with that given by a prism of dense flint glass having the same refracting angle. In the original drawing, $1 \mathrm{~mm}$. was taken to represent $1^{\prime}$ of arc.

It will be noticed that while piperine has almost exactly the same refractive power as dense flint glass for $\mathrm{H}_{y}$ in the blue, the refractive indices for rays of greater wave-length differ widely in the two substances, the result being that the visible spectrum given by piperine is nearly four times the length of that given by flint glass.

From the high refractivity of piperine, it seemed likely to be useful in the construction of certain kinds of polarising prisms. It would be 
hardly appropriate to enter into details here, but I may say that besides the usual type of nicol prism in which the extraordinary ray is transmitted while the ordinary ray is got rid of by total reflection, there is another class of polarising prisms made of Iceland spar, in which the ordinary ray is transmitted, the extraordinary ray being the one which is turned aside by total reflexion and abolished. For the latter class, of which the prisms devised by Jamin and by Bertrand may be taken as examples, a medium is required which shall have the same refractive index as that of Iceland spar for the ordinary ray, namely, 1.658. Now, although the refractivity of piperine is above this, it can easily be reduced to the proper level by admixture

Pipcrine.
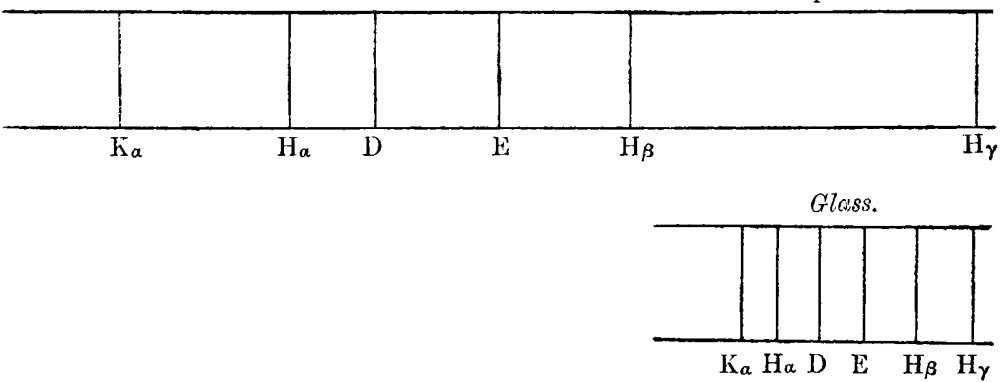

Comparative length of the spectre of piperine and glass.

with Canada balsam. In fact, I have a prism of this kind in which such a mixture (piperine, 4 parts; balsam, 1 part) replaces the usual balsam film. Unfortunately, however, owing to the extraordinarily high dispersive power of piperine, the critical angles for the different rays of the visual spectrum differ so widely that the prism is practically useless unless monochromatic light is employed.

Still, it would be easy to mention other purposes for which a medium of high dispersive power is useful, and it may be of some service to have called attention to the eminent degree in which piperine possesses this property. 